BULLETIN Bulletin hispanique

HISPANIQUE Université Michel de Montaigne Bordeaux

109-2 | 2007

La formation du Parnasse espagnol $X^{e}-X V I I{ }^{e}$ siècle

\title{
Francisco Rico, El texto del « Quijote». Preliminares a una ecdótica del Siglo de Oro
}

Anne Cayuela

\section{(2) OpenEdition}

1 Journals

Édition électronique

URL : http://journals.openedition.org/bulletinhispanique/387

DOI : 10.4000/bulletinhispanique.387

ISSN : 1775-3821

Éditeur

Presses universitaires de Bordeaux

Édition imprimée

Date de publication : 1 décembre 2007

Pagination : 749-756

ISBN : 978-2-85276-096-7

ISSN : 0007-4640

\section{Référence électronique}

Anne Cayuela, «Francisco Rico, El texto del « Quijote ». Preliminares a una ecdótica del Siglo de Oro », Bulletin hispanique [En ligne], 109-2 | 2007, mis en ligne le 10 février 2014, consulté le 21 septembre 2020. URL : http://journals.openedition.org/bulletinhispanique/387 ; DOI : https://doi.org/10.4000/ bulletinhispanique.387 


\section{COMPTE RENDU}

Francisco Rico, El texto del "Quijote ». Preliminares a una ecdótica del Siglo de Oro, Valladolid, Centro para la Edición de los Clásicos Españoles, Universidad de Valladolid, et Barcelone, Ediciones Destino, Biblioteca F.R., 2005, 568 p.

Si le Quichotte de Rico -c'est ainsi que les Cervantistes désignent son édition- est le texte dont le chef d'œuvre de Cervantès avait besoin, El texto del "Quijote", modestement qualifié de "préliminaires" à une ecdotique du Siècle d'Or représente l'aboutissement et le fruit de toute une réflexion théorique sur la science d'éditer les textes associée à une pratique magistrale. Dans son introduction, Francisco Rico déclare sans ambages son intention d'éditer les grandes œuvres du Siècle d'Or de la façon la plus rigoureuse et pertinente possible, intention qu'il a concrétisée à plusieurs reprises avec ses éditions des plus grands classiques de la littérature espagnole, Lazarillo de Tormes ${ }^{1}$, Guzmán de Alfarache ${ }^{2}$, La Celestina ${ }^{3}$, et plus récemment du Quichotte ${ }^{4}$. Francisco Rico rappelle que la connaissance des problèmes textuels est un préalable à toute étude sur le Quichotte, et consacre ce passionnant et brillant ouvrage à l'élucidation de ces questions. Aussi, au cours des 568 pages, il retrace toutes les étapes et les opérations successives

1. Anonyme, Lazarillo de Tormes, Barcelone, Planeta, 1983.

2. Mateo Alemán, Guzmán de Alfarache, Barcelone, Planeta, 1983.

3. Fernando de Rojas, La Celestina, Barcelone, Editorial Crítica, 2000.

4. Miguel de Cervantes, Don Quijote de la Mancha, Édition de l'Institut Cervantès, dirigée par Francisco Rico, Institut Cervantès- Crítica (Biblioteca clásica, 50), Barcelone, 1998 : un volume de texte (CCLXXXVI+1250 p.), et un volume complémentaire (1296 p.), plus un CD-ROM. La troisième édition corrigée (avril 1999) et ses réimpressions incluent toutes les corrections apportées au texte ainsi qu'à l'appareil critique depuis la première édition et sont recueillies sur la page web http://gould.uab.es/quijote:index.htlm. Plus récemment une nouvelle édition de l'Institut Cervantès 1605-2005 a été publiée par Galaxia Gutenberg, Círculo de Lectores y Centro para la Edición de los Clásicos Españoles, Barcelone, 2004: un volume de texte (CCCXXIV+1378 p.) plus un volume complémentaire (1456 p.), plus un CD-ROM.

BHi, Tome 109, nº 2 - décembre 2007 - p. 749 à 756. 
qui ont conduit à l'élaboration du texte imprimé du Quichotte. Si Cervantès introduit dans le livre lui-même le lieu et les opérations qui ont rendu possible sa publication "comment ici l'on tirait, là on corrigeait, là-bas on composait, ailleurs on révisait, avec tous les procédés qu’offrent les grandes imprimeries 5 ", et invite par là-même le lecteur à considérer la multiplicité des tâches nécessaires pour qu'un texte devienne un livre (la composition des pages par les compositeurs, la correction des premières feuilles imprimées à titre d'épreuves, la rectification des erreurs par les compositeurs dans les pages corrigées, et finalement l'impression des formes, -c'est-à-dire l'ensemble des pages destinées à être imprimées sur le même côté d'une feuille d'imprimerie, par les ouvriers en charge de la presse- ${ }^{6}$, Francisco Rico retrace avec minutie dans El texto del "Quijote" toutes ces opérations à l'intérieur de l'imprimerie, et y ajoute celles qui eurent lieu en amont et en aval telles que la correction du brouillon, les ajouts réalisés par Cervantès, ou les modifications introduites par la censure. Cette connaissance de la matérialité du livre ancien et des aspects techniques les plus élémentaires, indispensable pour comprendre certaines incohérences du texte, est associée à plusieurs disciplines telles que la philologie traditionnelle, l'Histoire du livre, ainsi que l'école angloaméricaine de la " editorial theory " et de la "textual bibliography " dont l'enseignement primordial est que « los usos y mańas de la imprenta manual con frecuencia determinan contra el deseo del autor la forma y el contenido textual de las obras de antaño $"{ }^{7}$. Ainsi, Francisco Rico explique qu'il s'agit de révéler, de faire émerger, de mettre au monde, un texte du Quichotte qui corresponde de la façon la plus précise à un texte "idéal " en se libérant de la tyrannie des éditions princeps, en cherchant de nouvelles directions, afin de résoudre le dilemme entre conserver ou corriger. Francisco Rico met l'accent sur la notion de " texte idéal » et se demande s'il s'agit de l'archétype, point de départ de tous les textes postérieurs, ou comme pour les textes de l'époque moderne, le texte d'arrivée issu des progressives révisions ou réélaborations auxquelles l'auteur l'a soumis (p. 246). Aussi propose-t-il une sorte de débat méthodologique autour d'une question centrale : comment

5. Michel de Cervantès, Lingénieux hidalgo Don Quichotte de la Manche, in Cervantès, Don Quichotte suivi de La Galatée, CEuvres romanesques, I, Edition publiée sous la direction de Jean Canavaggio, Paris, Gallimard, 2001, p.1357.

6. Cf. Roger Chartier : La presse et les fontes: Don Quichotte dans l'imprimerie, Conférence tenue par l'auteur lors de la deuxième session de l'École de l'Institut d'Histoire du livre, septembre 2002, http://ihl.enssib.fr/siteihl.php?page=165\&aflng=fr.

7. Francisco Rico, Imprenta y crítica textual en el Siglo de Oro, Valladolid, Centro para la Edición de los Clásicos Españoles, 2000, p. 9. 
doit-on employer les outils philologiques et historiques afin d'obtenir une édition critique satisfaisante pour la lecture du roman?

Francisco Rico rappelle dans son introduction que dans l'optique structuraliste chaque variante introduite dans un texte engendre un nouveau système, qui demande à être vu comme un ensemble auto-suffisant, comme une nouvelle rédaction. Dans la pratique la plus courante les variantes successives s'annulent entre elles pour déboucher sur un dernier et unique texte valable, alors qu'en réalité « cada edición, cada versión, es una realidad en plano de igualdad con las demás."(p. 304). L'auteur de El texto del "Quijote" n'hésite pas à manifester ses doutes et la perplexité théorique que suscite ce texte et ses variantes, perplexité qui débouche sur une réflexion qui va bien au-delà du texte de Cervantès: qu'est-ce qu'un texte ? Est-ce seulement chacune de ses réalisations concrètes, liées à des circonstances de production, un lieu et un temps, et à des codes bibliographiques, ou est-ce une abstraction de toutes ces réalisations concrètes ? Ce texte se trouve-t-il dans la tête de l'auteur, dans des signes graphiques, dans la perception du lecteur? (p. 307). Ce sont donc là des questions essentielles, auxquelles Rico sait ajouter une dose de mystère, attisant ainsi la curiosité du lecteur : à qui sont imputables les fameuses "erreurs ", les "oublis ", les "négligences " du texte ? Cervantès a-t-il corrigé certaines des éditions ? La solution est suggérée dès la page 45 : «De un lado está, convertido en poética del error voluntario, el dogma de la princeps (entiéndase : un facsimil de la princeps) y del nulo valor de la ediciones antiguas. Del otro lado, unos datos textuales y contextuales que responden a las prácticas y las concepciones de la época.» L'auteur révèle ainsi sa philosophie de l'édition critique, préconise la confrontation et l'examen minutieux du plus grand nombre possible d'éditions anciennes du texte et n'hésite pas à remettre en cause la méthode de certains chercheurs qui détaillent les variantes d'insignifiantes éditions du $\mathrm{XX}^{\mathrm{e}}$ siècle et ignorent la riche tradition des $\mathrm{XVII}^{\mathrm{e}}$ et $\mathrm{XVIII}{ }^{\mathrm{e}}$ siècles.

El texto del "Quijote" se compose d'un prologue, d'une introduction très substantielle du fait des questions théoriques soulevées, de 6 chapitres et de 6 articles joints en annexes ${ }^{8}$ à travers lesquels l'auteur de cet ouvrage magistral retrace tout le processus d'élaboration du texte de Cervantès. Francisco Rico choisit de mettre l'accent sur le travail des «hommes du livre" au temps de Cervantès, en particulier celui des correcteurs et des compositeurs « que poseían aún como suyas la lengua, la cultura y las formas de vida de Cervantes, y con ella una indudable capacidad para percibir

8. Publiés entre 1994 et 2005 ces articles constituent de véritables morceaux d'anthologie auxquels Rico renvoie dans le corps de son texte. 
problemas textuales que hoy, si no, se nos escaparían ». Cette présentation du processus d'édition du texte est toujours claire et précise, et les aspects les plus techniques sont expliqués et illustrés à grand renfort d'illustrations (82 au total), telles que les schémas qui permettent au lecteur de visualiser les cahiers de l'in-quarto et le pliage des feuilles, ou les reproductions de nombreux originaux d'imprimerie qui ont constitué une base documentaire exceptionnelle sur laquelle s'appuie la démonstration.

Au cours des 6 chapitres Francisco Rico met en lumière plusieurs points capitaux que nous signalerons ici :

Dans le premier chapitre, dont le titre « Cómo se hacía un libro en el Siglo de Oro " reprend celui d'un article déjà ancien de González de Amezúa, on voit que l'original d'imprimerie constitue un des jalons fondamentaux de ce passage du manuscrit à l'imprimé. L'atelier typographique ne travaillait pas à partir des manuscrits autographes -l'imprimerie de Juan de la Cuesta n'a jamais eu en sa possession le manuscrit autographe du Quichotte- mais à partir d'une transcription réalisée par un scribe engagé à cet effet. Cette copie portait le nom d' "original d'imprimerie " et c'est ce document que les auteurs revoyaient avec précision et non pas les épreuves. Grâce à ces précisions on voit combien le rôle du scribe est capital : "suele incrementar los yerros propios de toda transcripción con una irrestańable tendencia a la lectio facilior y la enmienda gratuita, y como trabaja deprisa, pues así se le pide que lo haga, y cobra por páginas, llega hasta henchirlas con tretas como doblar sistematicamente la ese intervocálica o preferir siempre sancto a santo (p. 69)».

Francisco Rico signale également le rôle du correcteur « un des principaux protagonistes de l'imprimerie au siècle d'or, et malheureusement, un des plus oubliés ». La mission du correcteur général était de vérifier que le corps de l'œuvre, qui lui parvenait une fois imprimée, correspondait à l'original visé par le Conseil et paraphé par un greffier. Contrairement à certains propos de spécialistes du Quichotte, la responsabilité de Murcia de la Llana en ce qui concerne les fautes du Quichotte fut nulle, car le rôle du correcteur général se limitait à signaler les différences entre l'original et le texte imprimé et n'intervenait pas sur le texte.

La connaissance de la technique de composition " par formes " permet de comprendre certaines modifications capitales entre le manuscrit autographe et le texte imprimé.

Le second chapitre « Del borrador a la censura » est consacré aux formalités administratives auxquelles était soumis le livre. Tandis que l'original (révisé ou non par l'auteur) était au Conseil, Cervantès introduisit dans le brouillon d'importants changements quant au contenu et à la structure du roman. 
Ceci expliquerait un certain nombre d'anomalies du roman telles que les épigraphes donnant pour accomplis des faits non narrés ou en annonçant d'autres déjà racontés, les disparitions et réapparitions de l'âne de Sancho, les repas servis deux fois, etc. Francisco Rico se demande comment Cervantès a procédé matériellement pour réécrire certains passages et les transférer d'un endroit à l'autre, quelles opérations concrètes il a réalisées dans divers manuscrits pour que la première rédaction devienne la deuxième et que demeurent des traces claires de la première. Il signale deux explications possibles aux modifications : celle imputables à la copie du copiste, celles dues au contrôle de la censure.

Le rôle-clef de l'imprimeur et certains aspects techniques de la réalisation matérielle du Quichotte fait l'objet du chapitre III intitulé "Por Juan de la Cuesta" selon la formule habituelle des frontispices des livres anciens. Rico s'intéresse au rôle de l'imprimeur et précise que la régularisation de la graphie et la ponctuation de l'original lui incombaient. Il pose au passage des questions essentielles comme la notion de "volonté de l'auteur ", de " texte autorisé ", et consacre ce chapitre à une véritable archéologie du livre, qui retrace avec une minutie remarquable la fabrication de l'édition de 1604.

Il révèle que la composition "par formes " est responsable de certaines variantes comme la suppression ou l'ajout de mots ou de phrases, démontrant ainsi la motivation purement typographique, étrangère à la volonté de l'auteur : « En cambio, la segunda y la tercera impresión del Ingenioso hidalgo nos garantizan que los operarios de Juan de la Cuesta, en más de un caso los mismos sin duda que en la primera, no tenían empacho en podar o injertar texto cuando les convenía para la composición por formas » (p. 192).

Le chapitre IV est consacré à la question des errata "plaie endémique en Espagne " et à la table des matières. On apprend que les éditions princeps du Quichotte ont souffert du manque de correction des épreuves et que si pendant le tirage d'une forme, on découvrait une faute, on interrompait l'impression, pour la corriger, et l'impression reprenait ensuite. A cause du prix élevé du papier les feuilles déjà imprimées n'étaient pas détruites, ce qui explique que de nombreux livres présentent deux ou trois états de certaines formes et qu'il est difficile de trouver deux exemplaires exactement identiques d'une même édition" (p. 212). On apprend également qu'une ligne mal justifiée, des blancs superflus ou n'importe quel autre détail qui enlaidissait l'apparence physique de la page était davantage un motif de préoccupation pour les imprimeurs qu'une confusion d'ordre sémantique (p. 214). Rico révèle également que la table des matières de 1604 n’a pas été établie à partir du texte imprimé mais à partir de l'original (p. 217) et que la table des matières n’a pas été élaborée par Cervantès. 
Enfin, le postulat, parfaitement démontré, concernant la composition de la princeps est le suivant : un original, soumis à révision au cours de laquelle Cervantès ouvrit de nouveaux chapitres, en supprima ou redistribua d'autres, réécrivit ou ajouta un certain nombre de passages (dans les marges, à la fin du manuscrit ou sur des feuilles ad hoc, en s'appuyant sur le brouillon, et même en ajoutant ou annexant à l'original des pages de ce brouillon. Et de conclure: "Las causas importan ahora menos en sí mismas que por las consecuencias que acarrean : pues, según se entiendan los epígrafes anómalos, habrá que admitirlos, suprimirlos o editarlos de una manera o de otra. No es un punto anecdótico, sino un problema ecdótico. "

Un important problème ecdotique est soulevé dans le chapitre V. Sous le titre de "El asno de Sancho " Francisco Rico s'interroge sur les ajouts des chapitres XXIII et du chapitre XXX dans l'édition de 1605, et démontre qu'ils sont l'œuvre de Cervantès.

Si l'intervention de l'éditeur Francisco de Robles ne fait aucun doute en ce qui concerne la seconde édition (p. 274) et si la grande majorité des variantes relèvent du correcteur, Cervantès est responsable d'un certain nombre d'entre elles (p. 276), en particulier les longs ajouts des chapitres XXIII et XXX, et les altérations qui affectent la structure globale du roman et le sens du texte de 1604, au point de conférer à l'édition de 1605 le statut de nouvelle rédaction, d'œuvre différente de l'édition princeps (p. 279). L'édition de 1608 trahit la volonté de l'auteur de retoucher le texte de façon plus ou moins semblable à celle de 1605 (p 282). Francisco Rico confère à l'édition bruxelloise de 1607 le titre de "bijou des premiers Quichottes", grâce à l'intervention du correcteur sur une multitude d'erreurs, au détriment d'une certaine authenticité typiquement cervantine (p. 283).

Enfin, dans le chapitre VI "Las huellas del rucio : cuestiones de principio » la disparition et les réapparitions de l'âne de Sancho font l'objet de brillantes analyses. Francisco Rico dévoile sa méthode pour traquer l'authenticité cervantine : l'analyse d'éléments vérifiables de façon objective comme les traits de langue et de style passés au crible de l'outil informatique; les facteurs contextuels qui postulent et délimitent la participation de l'auteur ; les passages épineux que la comparaison entre les variantes de la seconde et la troisième édition de l'Ingénieux hidalgo et celles des autres éditions anciennes met en lumière, les conditionnements typographiques, la censure inquisitoriale, les sources littéraires : c'est dire si les champs de compétence sont vastes et les outils affûtés. Nous indiquerons ici quelques-unes des conclusions de cette brillante démonstration :

Le texte du Quichotte a évolué depuis l'édition de 1604, il a été soumis à des ajouts et des retouches qui affectent la trame et ses articulations, déjà 
tournées vers les corrections de 1615. La seconde et la troisième édition ne sont pas les réimpressions " intellectuellement pirates » présupposées mais ont été contrôlées par Cervantès, et l'édition de 1605 a bénéficié d'une situation particulière : les deux longues interpolations des chapitres XXIII et XXX confèrent à la seconde édition la catégorie de rédaction, version ou refonte et la définissent comme un état distinct et autonome, dans lequel certains éléments changent de signification. La troisième édition est à considérer comme une "seconde édition de la seconde édition" car les retouches de 1608 se contentent de préciser les nouveautés apportées par l'édition de 1605 . Quant à l'édition de 1615 , elle suppose « un virage à $180^{\circ}$ ".

Francisco Rico décrit avec brio le long et complexe processus de composition du texte, les différents états qui ont supposé des réélaborations et des déplacements de texte, un mélange de divers états rédactionnels, une certaine confusion chronologique trahissant une "festiva despreocupación del relato por el raccord, por la continuidad de los detalles circunstanciales " (p. 296). La vision de l'œuvre littéraire qui en ressort est celle d' un processus vivant, soumis aux différentes lectures et malléable sous l'effet d'un dialogue constructif avec les lecteurs, " en una manera de colaboración social" ( $\mathrm{p}$. 306). L'auteur de El texto del "Quijote" annonce au passage le futur de l'édition critique : vouée à disparaître dans sa version imprimée, elle sera remplacée par des textes informatiques présentés sur le web et soumis à la continuelle actualisation des éditeurs et au questionnement des usagers ${ }^{9}$. Ainsi les nouveaux modèles de textualité suggérés par les possibilités de l'informatique engendreront aussi de nouveaux modèles de lecteurs.

Ces «préliminaires" à une ecdotique du Siècle d'Or s'avèrent être remarquablement aboutis. Francisco Rico nous livre conjointement une véritable théorie, une pratique et une philosophie de l'édition critique des classiques. «Editar un clásico entraña siempre un compromiso, un equilibrio entre las demandas del autor, del texto y del lector, y una apuesta del editor a la luz de unas convicciones teóricas o con ellas en la sombra " (p. 325). Il choisit de distinguer les variantes structurelles, qui révèlent deux ou même trois rédactions de la première partie, de variantes qui n'apportent pas de modifications infléchissant le cours de l'action et ne vont pas plus loin que le passage dans lequel elles interviennent. Il ne s'agit que de simples retouches de style ou de détail. Les variantes structurelles se distinguent donc des variantes ponctuelles mais forment un tout, un ensemble homogène à chaque étape. Selon Rico, le recours à un " texte de base " va à l'encontre de l'ecdotique : le travail de l'éditeur consiste à établir à travers ses corrections

9. Cf. note 4 . 
et ses interventions un texte plus fiable et le recours mécanique à un seul parmi les divers témoignages d'une œuvre équivaut à abdiquer du jugement critique justement là où il est le plus nécessaire. Il prône la reconnaissance de l'individualité historique de chaque version. (p. 328).

L'immense érudition, la qualité des citations, la connaissance de la bibliographie espagnole, française, italienne, anglo-saxonne, la qualité de la démonstration mais également la beauté de l'écriture -d'une précision et d'une élégance rares, non dépourvue d'un sens de la formule et d'un humour plein de malice-, tout concourt à faire de cet ouvrage un texte incontournable et passionnant pour tout philologue, qu'il soit simple lecteur, traducteur ou éditeur des grands textes de la littérature espagnole du Siècle d'Or.

Anne CAYUELA 